\title{
Impact of Congenital Cardiac Catheterization Project on Outcomes-Quality Improvement (C3PO-QI) in LMICs
}

\author{
Fatima Ali, ${ }^{1}$ Mohammad Qasim Mehdi, ${ }^{1}$ Saleem Akhtar, ${ }^{1}$ Nadeem Aslam, ${ }^{1}$ \\ Rashid Abbas, ${ }^{2}$ Izat Shah, ${ }^{2}$ Jabbir Abidi, ${ }^{2}$ Sajid Arthur, ${ }^{2}$ Zeenat Nizar, ${ }^{2}$ \\ Andrea Goodmann, ${ }^{3}$ Lisa Bergersen, ${ }^{3}$ Babar Hasan ${ }^{1}$
}

- Additional material is published online only. To view please visit the journal online (http://dx.doi.org/10.1136/ heartasia-2018-011105).

${ }^{1}$ Department of Pediatrics and Child Health, The Aga Khan University Hospital, Karachi, Pakistan

${ }^{2}$ Section of Cardiology, Department of Medicine, The Aga Khan University Hospital, Karachi, Pakistan ${ }^{3}$ Department of Cardiology, Boston Children's Hospital, Boston, Massachusetts, USA

\section{Correspondence to}

Dr Babar Hasan, Department of Pediatrics and Child Health, The Aga Khan University Hospital, Karachi 74800, Pakistan; babar. hasan@aku.edu

Received 11 August 2018 Revised 24 September 2018 Accepted 6 November 2018

\section{Check for updates}

(C) Author(s) (or their employer(s)) 2019. No commercial re-use. See rights and permissions. Published by BMJ.

To cite: Ali F, Qasim

Mehdi M, Akhtar S,

et al. Heart Asia

2019:11:e011105

doi:10.1136/

heartasia-2018-011105
ABSTRACT

Background The importance of registries for collaborative quality improvement has been overlooked in low/middle-income countries (LMIC). Aga Khan University Hospital (AKUH) in Pakistan joined the Congenital Cardiac Catheterization Project on OutcomesQuality Improvement (C3PO-QI) in March 2017 with the goal of leveraging international collaboration to improve patient care and institutional standards.

Methods The C3PO-QI key driver-based approach was used, with certain modifications, for process reengineering in AKUH's congenital cardiac catheterisation laboratory $(\mathrm{CCL})$ to reduce radiation exposure during cardiac catheterisation procedures (the primary outcome of (3PO- QI). Educating staff and standardising procedural documentation were the principal goals of the process re-engineering. Data survey was used to assess staff knowledge, attitude and practice before and after the initiative. Additionally, case demographics and outcomes were compared between AKUH and C3PO-QI centres.

Results There was an increase in appropriate recording of radiation surrogates $(0 \%-100 \%, p=0.00)$ and in the percentage of cases that met the established benchmark of 'Ideal documentation' (35\% vs $95 \%, p=0.001$ ). There was also an increase in self-reported staff interest during the case ( $25 \%$ vs $75 \%, p=0.001)$. AKUH versus C3PO-QI data showed similar demographic characteristics. There was a slight over-representation of diagnostic cases (42\% vs $32 \%$ ) as compared with interventional ( $58 \%$ vs $68 \%$ ) at AKUH. Furthermore, interventional procedures were predominately PDA and ASD device closures $(n=19$ and 15 , respectively). The frequency of adverse events were the same between AKUH and collaborative sites. Conclusion Collaborative efforts between developed and LMIC CCL are significant in advancing system-level processes.

\section{INTRODUCTION}

In cardiac catheterisation for congenital heart disease (CHD), a concerted effort is being made to transition from registries to quality improvement (QI) collaboratives. Such collaboratives have been shown to improve quality of care and help assess performance that is risk stratified for better comparisons. ${ }^{12}$ A classic example of such a transition is the Congenital Cardiac Catheterization Project on Outcomes (C3PO), a US-based multicentre registry launched in 2006 which assesses and compares outcomes while exploring measures

\section{Key messages}

What is already known about this subject?

- Collaborative quality assessment is a common practice in developed countries through the use of multicentre registries.

What does this study add?

- This will be the first report from a low/middleincome country (LMIC) on the impact of international collaboration and the resulting improvement of institutional practices in a lowresource setting.

How might this impact on clinical practice?

- By adopting the key driver diagram, any centre in an LMIC can evaluate and improve their radiation and procedural safety practices.

of procedural efficacy. ${ }^{3}$ In 2013, the C3PO collaborative moved beyond benchmarking and towards practice improvement through various QI initiatives (C3PO-QI) ${ }^{4-7}$ specifically reducing radiation exposure in paediatric cardiac catheterisation cases. ${ }^{8}$ Significant decreases in radiation exposure have resulted from this collaborative.

Experience from the International Quality Improvement Collaborative (IQIC) has also shown significant improvement in outcomes related to CHD surgeries in low/middle-income countries (LMIC), illustrating the success of collaboratives between developed countries and LMICs. ${ }^{9}$ A significant number of patients with CHD in LMICs who undergo CHD surgery also undergo cardiac catheterisation. The challenges in LMICs are unique and complex, including a lack of resources, late presentation of disease, inadequate skills and training, ${ }^{10} 11$ non-standardised documentation and a lack of system support. ${ }^{12-15}$ No registries or formal collaboratives exist to highlight these unique issues and their effects on procedural outcomes. Similarly, no collaboratives, such as those that exist for congenital cardiac surgery like the IQIC, have been established for the congenital cardiac catheterisation laboratory (CCL). The Aga Khan University Hospital (AKUH) joined the C3PO-QI as the first site from an LMIC, thus establishing the first such collaboration, in March 2017. The objective of this paper is (1) to demonstrate how association with a QI-based registry helps improve processes in CCL present in an LMIC and (2) to compare 


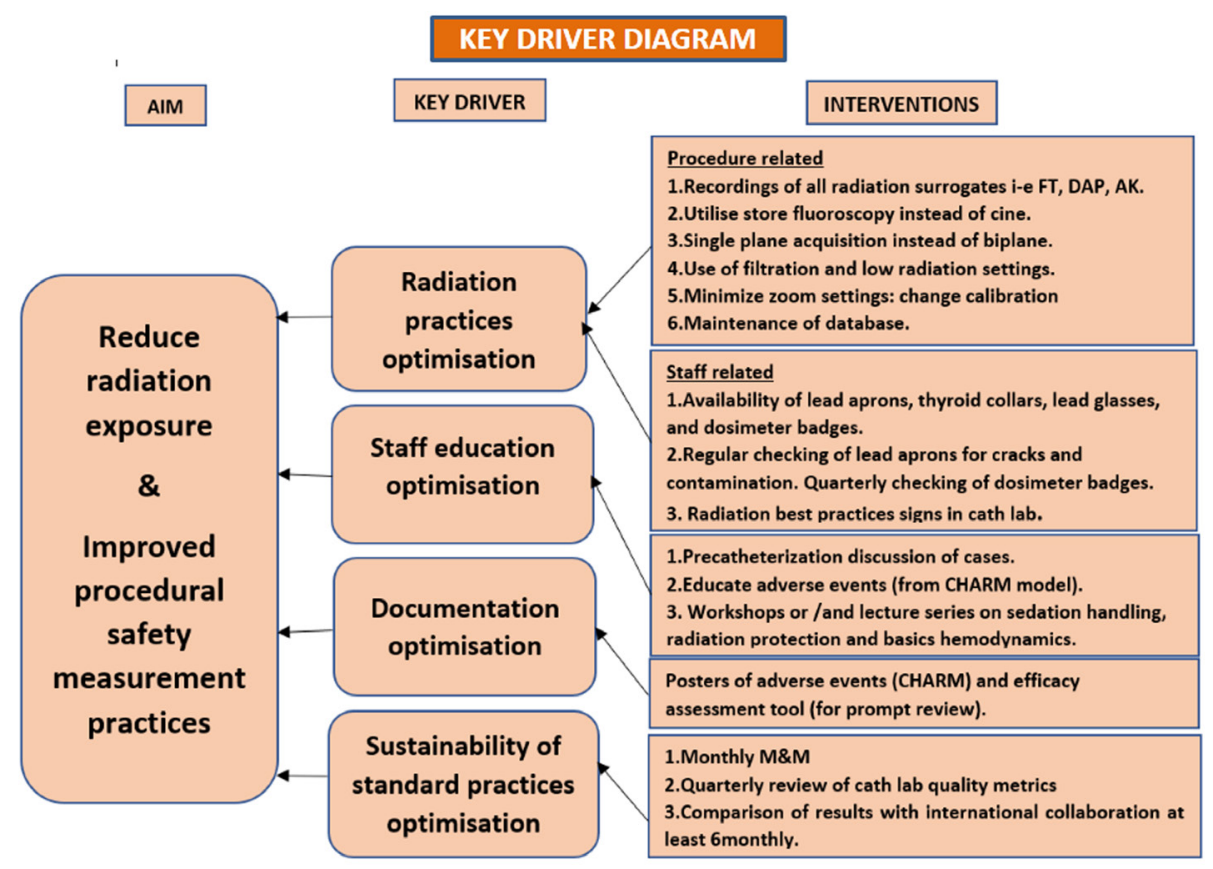

Figure 1 Key driver diagram with aim, key drivers and detailed interventions.

catheterization laboratory data at AKUH with the cumulative C3PO-QI participants.

\section{METHODS}

This is a comparative, cross-sectional descriptive study. This QI initiative project was conducted in the CCL of AKUH in Karachi, Pakistan, between September 2016 and November 2017 , and was divided into three phases. The preintervention phase (September 2016 to February 2017) was compared with a postintervention phase (June 2017 November 2017). Interventions took place between March 2017 to May 2017 (implementation phase).

\section{Identification of key drivers}

AKUH is a 644-bed tertiary care teaching hospital in Karachi, Pakistan, catering to all aspects of paediatric and adult diseases. The CCL has two single plane cameras and all resources (equipment and staff) are shared between paediatric and adult cases. Inappropriate radiation exposure was observed in congenital cardiac CT which led to an effort towards decreasing radiation exposure for CHD cases in any modality associated with radiation exposure. The principal investigator of C3PO-QI was contacted and AKUH joined the collaborative as its only international site in March 2017.

An internal audit of our system was performed to assess our readiness to join C3PO-QI. The following gaps were identified related to processes in our CCL pertaining to congenital cardiac catheterisations procedure:

1. Knowledge of radiation, its surrogates and efforts to reduce radiation exposure in the CCL were lacking. Only fluoroscopy time (FT) was used to track radiation. Standardised tracking of staff radiation exposure (proper dosimetry batch monitoring) and equipment radiation safety (lead apron cracks, availability of thyroid guards) were inadequate.

2. Inadequate procedure-related documentation, especially of adverse events and procedure efficacy assessment.
3. No standardised precase discussion that led to staff disinterest and a lack of case-related knowledge.

4. No quality metrics were tracked and the mortality/morbidity meetings were irregular.

Based on these factors, we identified the following four key drivers vital to improving safety practices:

1. Promotion of safe radiation practices.

2. Adequate staff education.

3. Documentation optimisation.

4. Sustainability of standard practices.

Interventions specifically targeting the above-mentioned key drivers were designed and executed (figure 1).

\section{Interventions}

An introductory lecture session was arranged for the CCL staff to introduce C3PO-QI, its objectives, data variables and the importance of joining the collaborative. This was done to familiarise the staff and get their buy-in by encouraging feedback and questions regarding the collaborative. The following specific interventions were then implemented (table 1).

\section{Data collection}

General variables that were collected included: age in years, gender, weight, height, diagnosis (physiological and STS), procedure type (diagnostic vs interventional), radiation surrogates (fluoroscopic time, air kerma, dose area products (DAP)) and details of adverse events, including categories (sedation or airway related, catheterisation related, access related, angioplasty, device, stent, coil, ablation, biopsy and valvuloplasty-related problems), event preventability (preventable, possibly preventable, not preventable) and event seriousness (levels 1-5).

\section{Assessment of the QI initiative}

The following outcome metrics were used to assess the interventions implemented for the described key drivers. 
Table 1 Key drivers with their specific interventions

\begin{tabular}{|c|c|}
\hline Key drivers & Interventions \\
\hline \multicolumn{2}{|l|}{$\begin{array}{l}\text { (A) Promotion of safe radiation } \\
\text { practices }\end{array}$} \\
\hline (1) Procedure related & $\begin{array}{l}\text { 1. Radiographers were educated on three radiation surrogates necessary to assessing procedural radiation exposure: fluoroscopy time (FT), dose area } \\
\text { product (DAP) and air kerma. A separate paediatric radiation register was also maintained for recording these values. } \\
\text { 2. Low radiation protocol such as low frame rates, stored fluoroscopy options, avoiding magnification and the use of filters was encouraged. Repeated } \\
\text { monitoring, immediate feedback and continual reinforcement of practices were implemented. } \\
\text { 3. Mandatory use of dosimeter badges for all staff involved in catheterisation procedures with check-ups every } 3 \text { months for radiation exposure (in } \\
\text { millisieverts (mSv)) was enforced. }\end{array}$ \\
\hline (2) Staff related & $\begin{array}{l}\text { 1. Use of appropriate protective gear was mandated and biannual checks for cracks and contamination were performed. } \\
\text { 2. Use of lead glasses, especially by primary operator, was encouraged. } \\
\text { 3. Radiation best practice signs were posted in the catheterisation lab. }\end{array}$ \\
\hline (B) Adequate staff education & $\begin{array}{l}\text { 1. Serial lectures to improve knowledge regarding cardiac haemodynamics, calculations, CHARM risk stratification and adverse events were initiated for } \\
\text { all CCL staff. This was done with the objective of raising staff awareness of patient status, recognising adverse event risk and detecting the beginnings } \\
\text { of an adverse event to enable timely response. } \\
\text { 2. Regular precatheterisation case discussions were initiated which included all the CCL staff (nurses, technicians, fellows, faculty, and so on). The purpose } \\
\text { was to increase interest and engagement of the staff during the procedure. This case discussion was brief and covered the diagnosis, the purpose of } \\
\text { the procedure, the risk category of the procedure, haemodynamics to expect, haemodynamic calculations and, for interventional cases, a discussion of } \\
\text { inventory and intervention sequence. The meeting ended with staff assignment of roles and responsibilities during the case. } \\
\text { 3. Staff engagement and interest were appreciated after procedure. } \\
\text { 4. The CCL staff was mandated to attend sedation and radiation protection workshops. } \\
\text { 5. All CCL staff members were involved in quality improvement projects in the lab. }\end{array}$ \\
\hline (C) Documentation optimisation & $\begin{array}{l}\text { 1. Catheterisation reports were standardised and all variables needed for } \mathrm{C} 3 \mathrm{PO}-\mathrm{Q} \text { I were captured. Documentation was deemed inadequate (as described } \\
\text { in table 2) if information was missing without a clear explanation. Immediate feedback was provided to the primary and secondary operators regarding } \\
\text { inadequate documentation. Addition of } \mathrm{PaO}_{2} \text { of oxygen for haemodynamic calculation was made essential during hyperoxia tests. } \\
\text { 2. A poster displaying pictures of procedural efficacy of the five interventions (patent ductus arteriosus (PDA), atrial septal defect (ASD) closure, } \\
\text { pulmonary and aortic valvuloplasties and coarctation of aorta ( } \mathrm{COA} \text { ) stenting) was placed in the } \mathrm{CCL} \text {. This was done to standardise procedural efficacy } \\
\text { documentation immediately during the case thus decreasing likelihood of any recall bias. }\end{array}$ \\
\hline $\begin{array}{l}\text { (D) Sustainability of standard } \\
\text { practices }\end{array}$ & $\begin{array}{l}\text { 1. A quality improvement team was formed including a head nurse, a radiographer, an interventional cardiologist, a cardiology fellow and the service chief. } \\
\text { This team was established to perform monthly audits in order to determine: the adequacy of documentation, effectiveness of morbidity and mortality } \\
\text { meetings, and staff radiation exposure using dosimetry batch data. The team also performed quarterly comparative analyses of AKUH versus cumulative } \\
\text { C3PO-QI data and biannually determined the adequacy of radiation protection gear. This team was also tasked with preparing quarterly reports on the CCL } \\
\text { quality metrics sharing the reports with the staff. }\end{array}$ \\
\hline
\end{tabular}

AKUH, Aga Khan University Hospital; CCL, cardiac catheterisation laboratory; CHARM, Catheterization for Congenital Heart Disease Adjustment for Risk Method; C3PO-QI, Congenital Cardiac Catheterization Project on Outcomes-Quality Improvement.

The following outcome metrics were used to assess the interventions implemented for the described key drivers: (1) documentation of radiation protection; (2) staff radiation protection; (3) operator techniques (for radiation practices optimisation); (4) self-interest and knowledge of CCL staff (staff education optimisation); and (5) catheterisation report documentation (documentation optimisation).

Outcomes were defined as adequate and inadequate as described in table 2 .

A questionnaire-based Knowledge, attitude, practices (KAP) survey (online supplementary appendix A) was conducted among 20 people, including nursing staff, technologist, cardiologists and trainee fellows. This survey assessed the knowledge and attitude regarding radiation surrogate, protection and information regarding congenital cardiac cases.

\section{Comparison of AKUH versus collective C3PO-QI cases}

Data from June 2017 to November 2017 of all patients with CHD who underwent cardiac catheterisation were entered into the C3PO-QI database. Variables that were used for comparison of AKUH versus C3PO-QI cumulative data included age $(<1$ year, 1-4 years, 5-9 years, 10-15 years and $>15$ years), case type (interventional vs diagnostic), radiation exposure (DAP/kg) and occurrence and severity of adverse events.

Various metrics (FT, air kerma, DAP, and so on) exist to determine the amount of radiation exposure during cardiac catheterisation. ${ }^{7}$ In pediatrics, the stochastic risk of radiation is the main concern rather than deterministic effects. ${ }^{4}$ DAP is the product of the air kerma dose and the cross-sectional area exposed to the X-ray beam $\left(\mu \mathrm{Gy}-\mathrm{M}^{2}\right){ }^{5}$ DAP provides a better

Table 2 Definitions (adequate vs inadequate) of outcome metrics

\begin{tabular}{|c|c|c|}
\hline Outcome metrics & Definition of adequate & Definition of inadequate \\
\hline $\begin{array}{l}\text { Documentation of radiation } \\
\text { surrogates }\end{array}$ & When all three indicators (FT, air kerma and DAP) were recorded. & When only one or two indicators were recorded. \\
\hline Operator technique & $\begin{array}{l}\text { When radiation protocols as low as reasonably achievable (ALARA), along with } \\
\text { appropriate use of cine imaging and magnification were used. }\end{array}$ & $\begin{array}{l}\text { High fixed frame rate imaging along with excessive cine imaging or } \\
\text { magnification was used. }\end{array}$ \\
\hline Staff radiation protection & $\begin{array}{l}\text { When lead aprons, thyroid collars and correctly positioned dosimeter badges were all } \\
\text { used. Quarterly check of dosimetry badges. }\end{array}$ & $\begin{array}{l}\text { When appropriate radiation protective gear was not used or dosimetry } \\
\text { badges not regularly checked. }\end{array}$ \\
\hline $\begin{array}{l}\text { Self-interest and knowledge } \\
\text { of CCL staff }\end{array}$ & $\begin{array}{l}\text { When the staff demonstrated understanding of case details such as diagnosis, the } \\
\text { purpose of the catheterisation, and possible adverse events and their management. } \\
\text { Additionally ability to perform accurate haemodynamic calculations. }\end{array}$ & $\begin{array}{l}\text { Lack of knowledge of case diagnosis, purpose of catheterisation and } \\
\text { possible adverse event. }\end{array}$ \\
\hline $\begin{array}{l}\text { Catheterisation report } \\
\text { documentation }\end{array}$ & $\begin{array}{l}\text { Documentation was considered ideal when all the variables required by the C3PO-QI } \\
\text { database were recorded. Documentation was considered adequate if it did not meet } \\
\text { the criteria for ideal or inadequate. }\end{array}$ & $\begin{array}{l}\text { In the absence of any of variables (diagnosis, access site, size } \\
\text { of devices, stent/balloon details and adverse event details), the } \\
\text { documentation was deemed inadequate. }\end{array}$ \\
\hline
\end{tabular}


Table 3 Demographics of cardiac catheterization cases performed at AKUH

\begin{tabular}{|c|c|}
\hline Variables ( $n=106$ patients) & n (\%) or median (IQR) \\
\hline \multicolumn{2}{|l|}{ Demographics } \\
\hline Age & $4(2.0,12)$ \\
\hline Weight (kg) & $14.0(7.0,29.5)$ \\
\hline Height (cm) & $98.0(71.5,141.0)$ \\
\hline Sex: female & $54(51)$ \\
\hline Physiologic diagnosis: no structural heart defects & $4(3.8)$ \\
\hline Isolated defects & $68(65)$ \\
\hline Pulmonary hypertension & $5(4.2)$ \\
\hline Complex two-ventricle physiology & $17(16)$ \\
\hline Single ventricle physiology & $12(11)$ \\
\hline \multicolumn{2}{|l|}{ STS diagnosis: thoracic artery defects } \\
\hline PDA & $21(20)$ \\
\hline $\mathrm{COA}$ & $4(3.8)$ \\
\hline \multicolumn{2}{|l|}{ Septal defects } \\
\hline ASD & $19(18)$ \\
\hline VSD & $18(17)$ \\
\hline CAVSD & $4(3.8)$ \\
\hline dTGA & $12(11.5)$ \\
\hline Single ventricle & $12(11.5)$ \\
\hline \multicolumn{2}{|l|}{ Right-sided lesions } \\
\hline PS & $5(4.5)$ \\
\hline Pulmonary atresia & $5(4.5)$ \\
\hline \multicolumn{2}{|l|}{ Left-sided lesions } \\
\hline AS & $1(1)$ \\
\hline Others & $5(4.5)$ \\
\hline Defined or suspected genetic syndrome & $5(5)$ \\
\hline \multicolumn{2}{|l|}{ Procedure type } \\
\hline Diagnostic & $45(43)$ \\
\hline All interventional & $61(58)$ \\
\hline \multicolumn{2}{|l|}{ Radiation } \\
\hline Fluoroscopy time (min) & $10.1(7.1,17.8)$ \\
\hline $\operatorname{DAP}(\mu \mathrm{Gy})$ & $163.5(85.9,465.3)$ \\
\hline Air kerma (mGy) & $21.5(11.4,53.5)$ \\
\hline Adverse event & $10(10)$ \\
\hline \multicolumn{2}{|l|}{ Categories } \\
\hline Sedation or airway related: apnoea & $1(1)$ \\
\hline \multicolumn{2}{|l|}{ Catheterisation-related problem } \\
\hline Arrhythmias & $2(2)$ \\
\hline Haemodynamics & $3(3)$ \\
\hline Others & $1(1)$ \\
\hline Access related: haematoma & $2(2)$ \\
\hline Stent-related problem: thrombosis & $1(1)$ \\
\hline \multicolumn{2}{|l|}{ Event } \\
\hline \multicolumn{2}{|l|}{ Preventability } \\
\hline Preventable & $4(4)$ \\
\hline Possibly preventable & $4(4)$ \\
\hline Not preventable & $2(2)$ \\
\hline \multicolumn{2}{|l|}{ Seriousness } \\
\hline Level 1 & $1(1)$ \\
\hline Level 2 & $3(3)$ \\
\hline Level 3 & $4(4)$ \\
\hline Level 4 & $2(2)$ \\
\hline
\end{tabular}

measure of the stochastic risk associated with cardiac catheterisation and has become a widely adopted method of radiation exposure reporting. ${ }^{48}$ When accounting for radiation, it is imperative to adjust for varying patient size and procedure type. Such risk adjustment provides an opportunity for fair comparison among centres and purposeful QI initiatives. The C3PO-QI recently showed that DAP indexed to weight (DAP/ $\mathrm{kg}$ ) provides an acceptable adjustment of outcomes, accounting for the variability in dose seen among cases of varying patient sizes. ${ }^{9}$ Similarly, risk stratification of radiation exposure based on procedural complexity and patient size has been proposed (manuscript in review) in which $90 \%$ of procedures performed in nine centres of the C3PO-QI collaborative were categorised into three radiation exposure categories: low $\left(<100 \mu \mathrm{Gy}-\mathrm{M}^{2} /\right.$ $\mathrm{kg})$, medium (100-200 $\left.\mu \mathrm{Gy}-3 \mathrm{M}^{2} / \mathrm{kg}\right)$ and high (>200 $\mu \mathrm{Gy}-\mathrm{M}^{2} /$ $\mathrm{kg}$ ). The radiation risk category type (rad type) was chosen for comparison in this manuscript as it incorporates both the procedural and patient size stratification.

Adverse events were broadly classified as problems related to the catheterisation, access, sedation or airway, angioplasty, device, stent, coil, ablation, biopsy or valvuloplasty. The following definitions were used for adverse event severity (online supplementary file 1$)^{5}$

he following definitions were used for preventability:

1. Not preventable: Any event in which the timing of the intervention cannot change the effect.

2. Possibly preventable: Any event in which timely intervention can change the effect.

3. Preventable: Any event missed and avoidable by appropriate care.

These data were captured from revised catheterisation reports and the paediatric radiation surrogate register. Data were entered by the senior fellow and internally audited twice by two cardiologists.

\section{Statistical analysis}

Simple frequencies were used for categorical data while $\chi^{2}$ test was used for comparison. For continuous variables, median with IQR was used. A p value $<0.05$ was considered significant.

\section{RESULTS}

A total of 106 congenital cardiac catheterisations were performed at AKUH. The median age of catheterisation was 4 $(2.0,12)$ years. Patent ductus arteriosus $(\mathrm{PDA} ; \mathrm{n}=21,20 \%)$ was the most common diagnosis followed by septal lesions (atrial septal defect (ASD) $n=19,18 \%$; ventricular septal defect $n=18$, 17\%) (table 3). The median FT was 10.1 min $(7.1,17.8)$, median DAP was $163.5 \mu \mathrm{g} / \mathrm{m}^{2}(85.9,465.3)$ and median air kerma was $21.5 \mathrm{mGy}(11.4,53.5)$. There was no mortality during the study period. Out of the $10(10 \%)$ morbidities, $4(4 \%)$ were preventable and an equal number were possibly preventable (table 3 ).

\section{Pre-C3PO-QI versus post-C3PO-QI: KAP survey}

Significant improvement in documentation of radiation surrogates (from $0 \%$ to $100 \%, \mathrm{p}=0.00$ ) and ideal procedural documentation (35\%-95\%, $\mathrm{p}=0.001)$ were observed. Marked change was observed in staff interest and knowledge $(25 \%-75 \%$, $\mathrm{p}=0.001$ ) of the case after implementation of precatheterisation discussion. Significant improvement was seen in adequate radiation selection technique $(29 \%-65 \%, \mathrm{p}=0.04)$ among the technicians while a trend towards improvement in adequate self-radiation protection practices by the staff $(41 \%-59 \%, \mathrm{p}=0.05)$ was observed. 


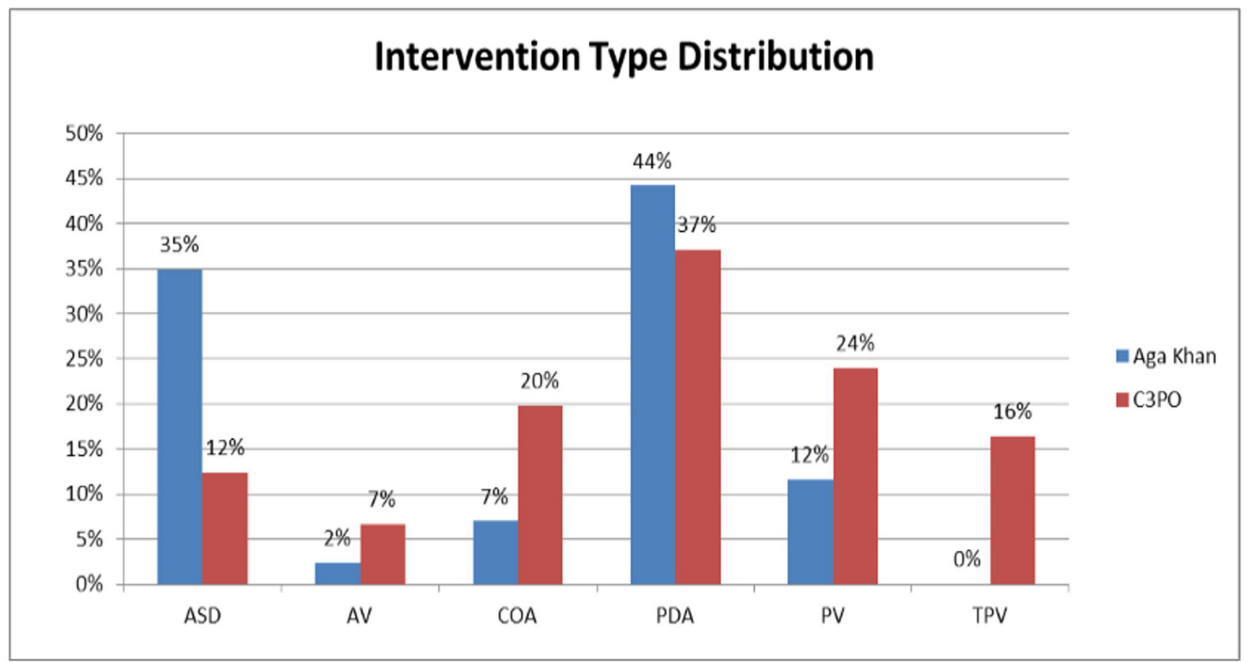

Figure 2 Aga Khan University Hospital (AKUH) versus Congenital Cardiac Catheterization Project on Outcomes-Quality Improvement (C3PO-QI) intervention type distribution comparison. ASD, atrial septal defect; AV, Aortic valvuloplasty; CoA, coarctation of aorta/dilation; PDA, patent ductus arteriosus; PV, Pulmonary valvuloplasty; TPV, Transcatheter pulmonary value replacement.

\section{AKUH versus C3PO-QI catheterisation data comparison}

A total of 106 cases were performed at AKUH compared with 2963 cases in the total C3PO-QI collaborative between 1 June and 31 November 2017. There was no difference between AKUH and the C3PO-QI collaborative in age distribution, with the majority of patients falling into the 1-4 years' age group (39\% vs 30\%). There was a slight over-representation of diagnostic cases (42\% vs 32\%) as compared with interventional $(58 \%$ vs $68 \%)$ at AKUH. PDA and ASD device closures $(n=19$ and 15 , respectively) were the predominant interventional procedures at $\mathrm{AKUH}$ as compared with PDA closure and pulmonary valve dilation in the C3PO-QI collaborative ( $\mathrm{n}=205$ and 131, respectively). Most notably, no TPV procedures were performed at our centre (figure 2).

The majority of cases at AKUH fell into the low and medium radiation risk category type with comparable radiation doses (figure 3).

Though radiation for interventional procedures was lower at AKUH, any conclusive interpretation is limited due to the small number of cases at AKUH (table 4).
Overall, AKUH had a lower incidence of adverse events than the collaborative ( $9 \%$ vs $11 \%$ ) though a higher proportion of high severity adverse events (level 3 and above) was seen at our centre (6\% vs 4\%) (online supplementary file 2 ).

The majority of adverse events were catheterisation-related problems (table 5) including arrhythmias and haemodynamics (table 3).

\section{DISCUSSION}

We showed that preparing to be a part of an international collaborative helps LMIC CCL programmes in system re-engineering which goes beyond the specific goals of the collaborative. Registries such as C3PO-QI allow for collaboration across institutions, provide large data sets for comparative assessments and improve the ability to generate outcome assessments. ${ }^{1}$ However, these registries are designed based on the US healthcare system and have, until now, never been implemented anywhere outside of the USA. Hence, the impact of such registries in an LMIC setting, with limited resources

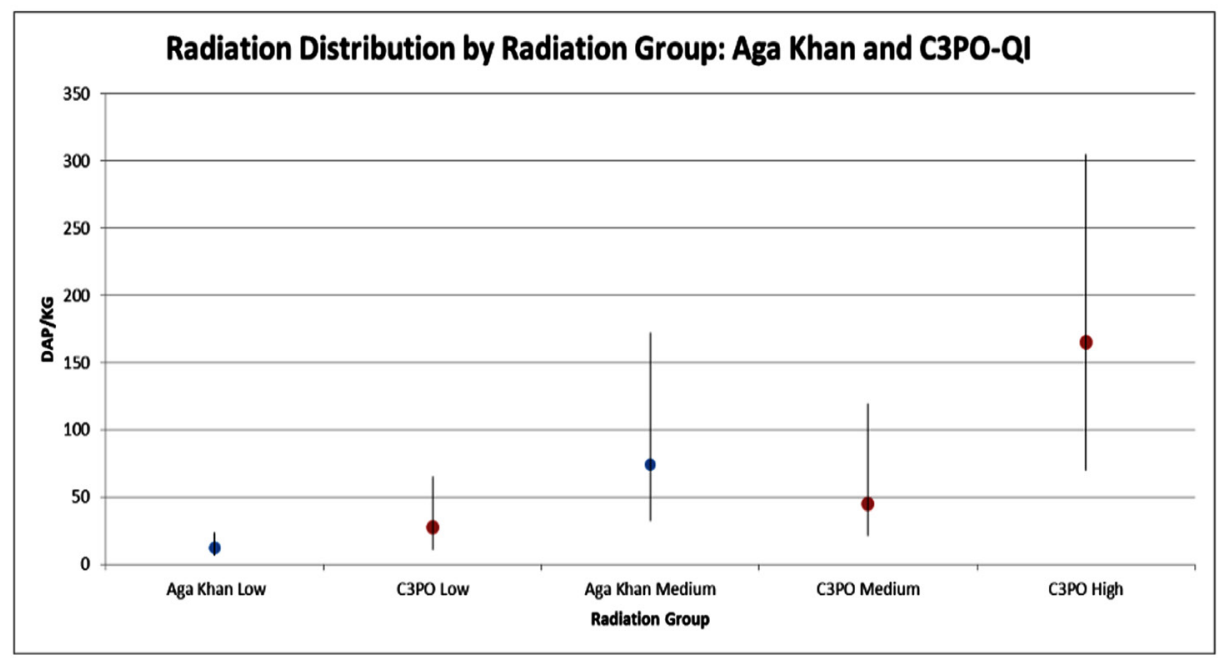

Figure 3 Aga Khan University Hospital (AKUH) versus C3PO-QI radiation distribution by radiation group (low, medium and high). C3PO-QI, Congenital Cardiac Catheterization Project on Outcomes-Quality Improvement; DAP, dose area product. 


\begin{tabular}{llrcr}
\hline $\begin{array}{l}\text { Table } 4 \\
\text { (DAP/kg) }\end{array}$ & AKUH versus C3PO-QI radiation exposure in interventions \\
\hline & $\begin{array}{l}\text { Aga Khan } \\
\text { Median (IQR), } \mathbf{n}\end{array}$ & \multicolumn{3}{l}{$\begin{array}{l}\text { C3PO } \\
\text { Median }(\text { IQR), } \mathbf{~}\end{array}$} \\
\hline ASD & $11.4(4.0,21.5)$ & 15 & $15.1(6.7,25.9)$ & 69 \\
\hline AV & 9.62 & 1 & $41.5(18.4,78.8)$ & 37 \\
CoA & $18.9(15.5,197.8)$ & 3 & $73.5(31.5,149.6)$ & 110 \\
\hline PDA & $9.4(74,24.8)$ & 19 & $20.7(9.7,38.1)$ & 205 \\
PV & $20.2(16.4,27.4)$ & 5 & $21.6(9.8,54.9)$ & 131 \\
\hline TPV & & 0 & $134.7(73.8,292.6)$ & 91 \\
\hline
\end{tabular}

AKUH, Aga Khan University Hospital; ASD, atrial septal defect; C3PO-QI, Congenital Cardiac Catheterization Project on Outcomes-Quality Improvement; CoA, coarctation of aorta; DAP, dose area product; PDA, patent ductus arteriosus.

and personnel, has never been assessed. To the best of our knowledge, this is the first report on the impact of such a collaborative in an LMIC on improving and maintaining the standards of congenital cardiac catheterisation procedures.

CHD possesses a significant health burden in LMICs like Pakistan, India and Indonesia. ${ }^{3} 16$ This is due to a higher birth rate, ${ }^{18}{ }^{19}$ late recognition and presentation of $\mathrm{CHD},{ }^{20-22}$ comorbidities like malnutrition and infections, ${ }^{17}$ lack of skills ${ }^{10}$ and the cost of treatment. ${ }^{10}$ Outcomes from CHD interventions (surgical or catheter based) in LMICs may differ from outcomes in developed countries due to these factors. ${ }^{23} 24$ There is a dearth of information regarding CHD interventions and their outcome from LMICs predominantly due to a lack of databases. This precludes LMIC CCL programmes from comparing outcomes, designing contextual risk stratification models and collaborating on QI. Lack of systematic data tracking and procedure standardisation in CCLs may also contribute to system inefficiency and poor outcomes. ${ }^{1}$ Like other CCLs in LMICs, the experience at AKUH prior to joining the collaborative demonstrated several system-level issues including inadequate documentation, inadequate tracking of procedural outcomes, lack of staff interest in congenital cases and improper radiation safety measures (both for patient and staff). ${ }^{25-28}$ The success of IQIC in highlighting CHD surgeries in LMICs and significantly improving postoperative morbidity and mortality in patients with CHD is an excellent example of the effectiveness of collaboration between developed and LMIC CHD programmes. ${ }^{9}$ System-level changes (nurse empowerment, QI teams, systematic data tracking, and so on) and improved practices (surgical checklist, infection control, and so on) were some of the key factors in determining the

\begin{tabular}{|c|c|c|}
\hline Adverse event categories & $\begin{array}{l}\text { Aga Khan } \\
\text { n (\%) }\end{array}$ & $\begin{array}{l}\text { C3PO-QI } \\
\text { n (\%) }\end{array}$ \\
\hline Catheterisation-related problem & $6(6)$ & $134(40)$ \\
\hline Access-related problem & $2(2)$ & $90(27)$ \\
\hline Sedation or airway-related problem & $1(1)$ & $33(10)$ \\
\hline Angioplasty-related problem & $0(0)$ & $28(8)$ \\
\hline Device-related problem & $0(0)$ & $15(5)$ \\
\hline Stent-related problem & $1(1)$ & $17(5)$ \\
\hline Coil-related problem & $0(0)$ & $8(2)$ \\
\hline Ablation-related problem & $0(0)$ & $1(<1)$ \\
\hline Biopsy-related problem & $0(0)$ & $1(<1)$ \\
\hline Valvuloplasty-related problem & $0(0)$ & $5(2)$ \\
\hline
\end{tabular}

AKUH, Aga Khan University Hospital; C3PO-QI, Congenital Cardiac Catheterization Project on Outcomes-Quality Improvement. success of IQIC in our CHD surgical programme. ${ }^{9}$ We joined C3PO-QI with the intention of not just targeting radiation improvement but to bring about a system-level change in the CCL. This was successfully demonstrated in the improvement and standardisation of documentation, radiation control practices and, most importantly, better staff engagement. Better staff emotional engagement has been demonstrated to be the most important factor in patient experience of care $^{29}$ and, in turn, is associated with higher quality and safer care.

Though the numbers are limited for any conclusive interpretation, it is interesting to note that the age and case distributions were similar between our centre and the collaborative. Similar adverse outcomes were noticed, with catheterisation-related complications being the highest. Thrombosis of PDA stents contributed to the worse adverse events at AKUH. Our centre did not perform any TPV (due to the cost of the device) and thus did not have any high radiation category procedures. Among the medium and low radiation procedure categories, our results were comparable to the collaborative. Interestingly, ASD, PDA and CoA had much lower radiation exposure in our setting compared with the collaborative. This may be due to use of single plane imaging during the procedure compared with biplane imaging in CCLs of the collaborative.

\section{Limitations}

As a single centre study, the generalisability of our results is limited to our locale and similar resource-limited settings. The comparison of preintervention versus postintervention self-proclaimed knowledge acquisition surveys was conducted in the immediate period after intervention. It is well known that this is the greatest period of knowledge retention thus more useful assessment of knowledge retention is planned. Additionally, small number of staff (though all of our CCL staff were interviewed) may impair conclusive interpretation. Though the similarity in case distribution and outcomes and differences in radiation doses were interesting, the small number of cases from our centre may preclude any significant interpretation of these results. It will be interesting to see how these results sustain over time as the number of cases entered increases.

\section{CONCLUSION}

Collaborative efforts between developed and LMIC CCL are helpful in significant improvement among system-level processes. The benefit of such a collaborative, thus, is above and beyond just the specific outcome targeted by the collaborative, that is, improvement in radiation, and in fact participation in the collaborative helps CCLs in LMIC to bring about system-level changes.

Contributors FA and BH have contributed equally to the study concept, designing, data collection, analysis and manuscript writing. MQM contributed to the data collection, data analysis and manuscript writing. SA and NA helped in data analysis and manuscript writing. RA, IS, JA, SA and ZN contributed to data collection and data analysis. AG and LB helped in critical designing and revision of the manuscript. All authors have contributed significantly and agree with the content of the manuscript.

Funding The authors have not declared a specific grant for this research from any funding agency in the public, commercial or not-for-profit sectors.

Competing interests None declared.

Patient consent Not required.

Provenance and peer review Not commissioned; externally peer reviewed. 


\section{REFERENCES}

1. Jenkins KJ, Beekman lii RH, Bergersen LJ, et al. Databases for assessing the outcomes of the treatment of patients with congenital and paediatric cardiac disease--the perspective of cardiology. Cardiol Young 2008;18:116-23.

2. Vener DF, Gaies M, Jacobs JP, et al. Clinical databases and registries in congenital and pediatric cardiac surgery, cardiology, critical care, and anesthesiology worldwide. World J Pediatr Congenit Heart Surg 2017;8:77-87.

3. Chaudhry-Waterman N, Coombs S, Porras D, et al. Developing tools to measure quality in congenital catheterization and interventions: the congenital cardiac catheterization project on outcomes (C3PO). Methodist Debakey Cardiovasc J 2014; 10:63-7.

4. Cevallos PC, Armstrong AK, Glatz AC, et al. Radiation dose benchmarks in pediatric cardiac catheterization: A prospective multi-center C3PO-QI study. Catheter Cardiovasc Interv 2017;90:269-80.

5. Bergersen L, Gauvreau K, Foerster SR, et al. Catheterization for congenital heart disease adjustment for risk method (CHARM). JACC Cardiovasc Interv 2011:4:1037-46.

6. Jayaram N. Beekman RH 3rd, Benson L, et al. Adjusting for Risk Associated with Pediatric and Congenital Cardiac Catheterization: A Report from the NCDR IMPACT TM Registry. Circ 2015;132:1863-70.

7. Cevallos PC, Rose MJ, Armsby LB, et al. Implementation of Methodology for Quality Improvement in Pediatric Cardiac Catheterization: A Multi-center Initiative by the Congenital Cardiac Catheterization Project on Outcomes-Quality Improvement (C3PO-QI). Pediatr Cardiol 2016;37:1436-45

8. Ghelani SJ, Glatz AC, David S, et al. Radiation dose benchmarks during cardiac catheterization for congenital heart disease in the United States. JACC CardiovasC Interv 2014;7:1060-9.

9. Khan $A$, Abdullah $A$, Ahmad $H$, et al. Impact of International quality improvement collaborative on congenital heart surgery in Pakistan. Heart 2017;103:1680-6.

10. Kumar RK, Tynan MJ. Catheter interventions for congenital heart disease in third World countries. Pediatr Cardiol 2005;26:241-9.

11. Brickner ME, Hillis LD, Lange RA. Congenital heart disease in adults. N Eng/ J Med Overseas Ed 2000;342:256-63.

12. Kumar RK, Shrivastava S. Paediatric heart care in India. Heart 2008:94:984-90.

13. Kumar RK, Tynan MJ. Catheter interventions for congenital heart disease in third world countries. Pediatr Cardiol 2005;26:241-9.

14. Lock JE, Keane JF, Perry SB. Diagnostic and interventional catheterization in congenita heart disease. Springer Science \& Business Media 2000
15. National Research Council. Health risks from exposure to low levels of ionizing radiation: BEIR VII phase 2. National Academies Press, 2006.

16. van der Linde $D$, Konings EE, Slager MA, et al. Birth prevalence of congenital heart disease worldwide: a systematic review and meta-analysis. J Am Coll Cardiol 2011;58:2241-7.

17. Kumar RK. Screening for congenital heart disease in India: Rational, practica challenges, and pragmatic strategies. Ann Pediatr Cardiol 2016;9:203-4

18. Nguyen $\mathrm{N}$, Leon-Wyss J, lyer KS, et al. Paediatric cardiac surgery in low-income and middle-income countries: a continuing challenge. Arch Dis Child 2015;100:1156-9.

19. Hoffman Jle. The global burden of congenital heart disease. Cardiovasc J Afr 2013;24:141-5.

20. Bernier PL, Stefanescu A, Samoukovic G, et al. The challenge of congenital heart disease worldwide: epidemiologic and demographic facts. Semin Thorac Cardiovasc Surg Pediatr Card Surg Annu 2010;13:26-34.

21. Hoffman JI, Kaplan S. The incidence of congenital heart disease. J Am Coll Cardiol 2002;39:1890-900.

22. Deanfield J, Thaulow E, Warnes C. Task Force on the Management of Grown Up Congenital Heart Disease. European Society of Cardiology. Eur Heart J 2005:24:1035-84

23. Putra ST, Djer MM, Idris NS, et al. Transcatheter closure of atrial septal defects in a center with limited resources: outcomes and short term follow-up. Iran J Pediatr 2015;25:e3906.

24. Vitiello R, McCrindle BW, Nykanen D, et al. Complications associated with pediatric cardiac catheterization. J Am Coll Cardiol 1998;32:1433-40.

25. Balachandran R, Kappanayil M, Sen AC, et al. Impact of the International Quality Improvement Collaborative on outcomes after congenital heart surgery: a single center experience in a developing economy. Ann Card Anaesth 2015;18:52-7.

26. Verghese GR, McElhinney DB, Strauss KJ, et al. Characterization of radiation exposure and effect of a radiation monitoring policy in a large volume pediatric cardiac catheterization lab. Catheter Cardiovasc Interv 2012;79:294-301.

27. Hill KD, Frush DP, Han BK, et al. Radiation safety in children with congenital and acquired heart disease: a scientific position statement on multimodality dose optimization from the image gently alliance. JACC Cardiovasc Imaging 2017; 10:797-818

28. Justino $H$. The ALARA concept in pediatric cardiac catheterization: techniques and tactics for managing radiation dose. Pediatr Radio/ 2006;36:146-53.

29. Sisneros D. Employee Engagement: Key to the Exceptional Patient Experience. Language of Caring 2014;6:1-9. 